Punjab University Journal of Mathematics (2021),53(8),557-573

https://doi.org/10.52280/pujm.2021.530803

\title{
Numerical Solution of Time Fractional Delay Partial Differential Equations by Perturbation Iteration Algorithm
}

\author{
Fareeha Sami Khan ${ }^{a}$, Mariam Sultana ${ }^{b}$ and M. Khalid ${ }^{c}$ \\ ${ }^{a, b, c}$ Department of Mathematical Sciences, \\ Federal Urdu University of Arts, Sciences \& Technology, Karachi-75300, Pakistan. \\ Email: ${ }^{a}$ fareeha.samikhan@fuuast.edu.pk, ${ }^{b}$ marium.sultana@ fuuast.edu.pk, \\ ${ }^{c}$ khalidsiddiqui@fuuast.edu.pk
}

Received: 02 February, 2021/ Accepted: 09 August, 2021/ Published online: 26 August, 2021

\begin{abstract}
The aim of this research was to relate two physical effects for partial differential equations on the time-coordinate, notably the multipledelay times and fractional-derivative. Time Fractional Delay Partial Differential Equations (TFDPDEs) usually interpret some complex physical phenomenon. This study works to solve TFDPDE with shrinking in $x$ and proportional delays in $t$ numerically by utilizing the fractional derivative of Caputo sense in the numerical method known as Perturbation Iteration Algorithm (PIA). A few famous numerical examples have been solved using PIA and their comparison with an exact solutions is illustrated for $\alpha=1$. Also, different values of $\alpha$ have been depicted in graphical form to show their fractional behavior. The delay term $k$ is also discussed extensively in this TFDPDE study. Numerical results show that this technique is reliable, convenient, and attractive for computational use in modern times.
\end{abstract}

AMS Subject Classification Codes: 47A55; 26A33; 35R10; 34K37; 35C10; 97Nxx Key Words: Partial Differential Equations, Fractional derivative of Caputo, Perturbation Iteration Algorithm, Numerical method, Exact solutions.

\section{INTRODUCTION}

Fractional calculus is as long established as the theory of integer calculus. Recently, it has started attracting the interest of researchers due to its vast and deep understanding of real life phenomena described through mathematical models. Fractional calculus can be understood as the generalization of classical calculus, as it analyses complex and real numbers as orders of derivatives. Lately, numerous researchers have studied physical phenomena using fractional derivatives; for instance, epidemiological models, signal processing, 
fluid mechanics, diffusion reaction process, electrical networks, dengue fever, HIV/Aids model etc. [19,21].

Indeed, to obtain the solution which is exact of any extensive class of differential equation cannot be effortless. Keeping all this in mind, many researchers have developed different kinds of robust techniques to find an approximate solution of such complicated type of fractional differential equations by using different versions of power series method, perturbation method, decomposition method and finite difference method.

All of the above techniques contributed a whole lot in understanding the fractional behavior of various physical phenomenons. Above discussed mathematical methods have also made valuable contributions to the analysis of Partial Differential Equations (PDEs) arising in many problems of science and engineering. Generalized types of classical PDEs, also commonly known as fractional order PDEs, also have implementations in diverse fields such as hydrology, fluid flow, transport processes having long term memory, finance etc. $[11,26,28]$. Such kind of equations also have applications in medicine and biology, as well as in population and environmental ecology. These equations may also be used in control system models and climate models etc. [29]. Their one or more than one dimension variables are denoted by $x$ and their independent variables are time $t$, which generally represents the position, length, or rate of maturation in cell space etc. The solutions can reflect different particle voltage, temperature, and size on fractional time intervals. The core work of PDE problems is to relate an unknown function to its evaluated partial derivatives at the same time.

Conventional instantaneous differential equations cannot reveal as good a variety of temporal spatial patterns as Delay Partial Differential Equations (DPDEs). Such DPDEs in many scientific fields provide more reasonable models for phenomena that show time lags or memory effects. These DPDEs have applications in generic repression [9, 23] (considering the reactant's spatial diffusion in the models and time delays from processes of translation and transcription), population ecology $[11,25]$ (to analyse the interconnection among time delays and spatial diffusion), modeling cell division and cell growth depending on its size $[8,20]$ and general control problems [13] ( cases in which time delay occurs due to the problems in information processing in transmission etc). In short, delay partial differential equations (DPDEs), and Time-Fractional Delay Partial Differential Equations (TFDPDEs) not only model physical problems that depend on the current state of the system, but also on past history. Several such examples can be seen in recent literature as $[1-7,14]$. They solved fractional delay differential equations like Newell- Whitehead-Segel system, fractional delay type wave equation, fractional Boussinesq-Burgers equation, fractional twomode Korteweg-De Vries equation, fractional reaction-Diffusion Brusselator model, and Duffing equation by many interesting techniques and obtained several fascinating results. Till date the theory of PDEs with delay or fractional dynamic systems with delay is developed comprising of observations of existence, uniqueness of their solutions and stability of its solutions as well as equilibrium states. Such as the stability and uniqueness of PDEs with nonlinear fractional term is studied by [24]. By using Banach contraction mapping theorem, Lebesgue dominated convergence theorem and the fixed point theorem by Leray-Schuader, [24] derived the necessary and sufficient conditions for the existence of a category of equations. [30] introduced a fractional order model of CD4 ${ }^{+}$T-cell HIV infection, obtained the equilibrium points with infected and non infected states and also proved 
the stability of these equilibrium points. Also [31] proved the stability and developed the sufficient and necessary conditions for a linear time fractional delay system. Since we all understand, solving FPDEs effectively and precisely with delay is a mathematically challenging task.

These TFDPDEs can also yield reasonable and realistic results if they are solved with the numerical methods described above. Homotopy Perturbation method is applied on Fractional Fornberg Whitham equation to analyze the numerical solution of Fractional Fornberg Whitham equation by [12]. Linearised finite difference scheme was applied on convection diffusion equation of fractional order by using the reversible exponential recovery method by [27]. In [22] homotopy perturbation method is applied for solving the non linear fractional PDEs with proportional delays numerically. Also in [10], authors used VIM to solve fractional partial delay differential equations numerically.

Sometimes the calculation effort is immense and therefore the mechanism of computation becomes significantly more difficult if solved by these numerical techniques. Among them is a method named Perturbation Iteration Algorithm (PIA), in this paper three TFDPDEs are solved numerically by PIA to show the efficacy of this method and how effortless is the calculation process by PIA.

PIA has proven to be effective in solving almost all type of differential equations [15], partial differential equation [16], fractional system of differential equation [17] , fractional differential difference equation [18], etc. Now in this paper, the efficacy of PIA will be proven for TFDPDEs.

The paper is divided into the following sections: Section I gives a brief literature review. Section II is about the preliminary concepts of fractional calculus, Section III is about the development of mathematical theory of PIA for solving TFDPDEs. Section IV discusses the applicability of PIA on three different examples of TFDPDEs and their graphical illustration of comparison among exact and the numerical solution as well as for different values of $\alpha$. Section $\mathrm{V}$ is the conclusion drawn from this work.

\section{Preliminary Concepts of Fractional Calculus}

Some fundamental concepts of the fractional calculus used mostly for solving TFDPDEs in this paper are given with just a brief overview:

Definition 2.1. [25] Caputo fractional order derivative with order $\alpha$, which is used often is written as

$$
D_{t}^{\alpha} v(t)=\frac{1}{\Gamma(n-\alpha)} \int_{0}^{t}(t-\tau)^{n-\alpha-1} v^{(n)}(\tau) d \tau
$$

where $n-1<\alpha \leq n, n \in N, t>0$.

For $\alpha=1$ Caputo sense derivative becomes

$$
D^{\alpha} v(t)=\frac{d v(t)}{d t}
$$

2.2. Properties of Caputo Fractional Derivative. [25] Some properties of Caputo derivative used in this work are

(a) $D_{t}^{\alpha} t^{\gamma}=\frac{\Gamma(1+\gamma)}{\Gamma(1+\gamma-\alpha)} t^{\gamma-\alpha} ; \quad \gamma>0$ 
(b) $D_{t}^{\alpha}(c v(t))=c D_{t}^{\alpha} v(t)$, where c is constant.

(c) $D_{t}^{\alpha}(a v(t)+b u(t))=a D_{t}^{\alpha} v(t)+b D_{t}^{\alpha} u(t)$, where a and b are constant.

(d) $D_{t}^{\alpha} c=0$.

\section{MATHEMATICAL FORMULATION}

Perturbation Iteration Algorithm is a Taylor-series-based method that involves a small perturbation parameter $\epsilon$ for eliminating the non linear terms by formulating the iterative expansion. Further, by using the usual methods, the Time fractional Delay Partial Differential Equation is solved. The steps of Perturbation Iteration Algorithm for solving TFDPDE are given below.

Consider the general form of TFDPDE be written as

$$
F_{t}^{\alpha} v(x, t)=g\left(x, t, v\left(k_{\circ} x, k_{\circ} t\right), F_{x} v\left(k_{1} x, k_{1} t\right), \cdots, F_{x}^{n} v\left(k_{n} x, k_{n} t\right)\right) ; \quad n=0,1,2, \cdots
$$

Subject to initial condition $F^{q} v(x, 0)=C_{q}(x)$ for $k=0,1,2, \cdots, m<\alpha \leq m+1$, $m \in R$ that describes the order of time fractional derivative, where $(x, t) \in[0,1] \times[0,1]$, is a specified initial function, $t$ and $x$ are independent variables, $k_{i}, k_{j} \in(0,1)$ for $i, j \in R$ and $v(x, t)$ is the unknown function.

PIA(1,1) denotes the expansion of Taylor series and correction terms up to first order derivatives only. In this paper PIA $(1,1)$ will be used from PIA(m,n) only. Now, consider that the system has the following solution

$$
v_{q, r+1}=v_{q, r}+\epsilon v_{q, r}^{c}
$$

In Eq.(3.4), for perturbation expansion, $v_{q, r}^{c}$ represents the correction term and $r$ as subscript means the $r^{\text {th }}$ number of iteration of this approximate solution. Now by expanding Eq.(3.3) by Taylor series in the neighborhood of $\epsilon=0$ it becomes

$$
P=\sum_{m=0}^{M} \frac{1}{m !}\left[\left(\frac{d}{d \epsilon}\right)^{m} P\right]_{\epsilon=0} \times \epsilon^{m}
$$

where $\frac{d}{d \epsilon}$ is defined as

$$
\frac{d}{d \epsilon}=\frac{\partial v_{q, r}}{\partial \epsilon} \frac{\partial}{\partial v_{q, r}}+\sum_{p=1}^{q}\left(\frac{\partial v_{q}}{\partial \epsilon} \frac{\partial}{\partial v_{q}}\right)+\frac{\partial}{\partial \epsilon}
$$

Combining Eq.(3.5) and Eq.(3.6), the following equation becomes the iterative equation

$$
P=\sum_{m=0}^{M} \frac{1}{m !}\left[\left(\frac{d}{d \epsilon}=\frac{\partial v_{q, r}}{\partial \epsilon} \frac{\partial}{\partial v_{q, r}}+\sum_{p=1}^{q}\left(\frac{\partial v_{q}}{\partial \epsilon} \frac{\partial}{\partial v_{q}}\right)+\frac{\partial}{\partial \epsilon}\right)^{m} P\right]_{\epsilon=0} \times \epsilon^{m}
$$

Upon expansion the terms of Eq.(3.7) is written in the form

$$
\begin{aligned}
& g\left(x, t, v\left(k_{\circ} x, k_{\circ} t\right), F_{x} v\left(k_{1} x, k_{1} t\right), \cdots, F_{x}^{n} v\left(k_{n} x, k_{n} t\right), 0\right)+g_{x}\left(x, t, v\left(k_{\circ} x, k_{\circ} t\right),\right. \\
& \left.F_{x} v\left(k_{1} x, k_{1} t\right), \cdots, F_{x}^{n} v\left(k_{n} x, k_{n} t\right), 0\right)\left(F_{x}\right)_{n}^{c} \epsilon+g_{x x}\left(x, t, v\left(k_{\circ} x, k_{\circ} t\right), F_{x} v\left(k_{1} x, k_{1} t\right), \cdots,\right.
\end{aligned}
$$




$$
\left.F_{x}^{n} v\left(k_{n} x, k_{n} t\right), 0\right)\left(F_{x x}\right)_{n}^{c} \epsilon+\cdots+g_{\epsilon} \epsilon=0
$$

After a little simplification it becomes as

$$
\left(F_{x^{n}}^{c}\right)_{n}+\frac{g_{x^{n-1}}}{g_{x^{n}}}\left(F_{x^{n-1}}^{c}\right)_{n}+\cdots=-\frac{g_{\epsilon}+\frac{g}{\epsilon}}{g_{x^{n}}}
$$

Now, Eq.(3.9) is said to be the linear time fractional delay partial differential equation. If the initial condition is applied on Eq.(3.9), the remaining terms will only be the correction terms. Also remember all these calculations are preferred to solve for $\epsilon=0$ only

$$
\left(F_{t}^{\alpha} v(x, t)\right)^{c}=-F_{t}^{\alpha} v(x, t)+L\left(g\left(x, t, v\left(k_{\circ} x, k_{\circ} t\right), F_{x} v\left(k_{1} x, k_{1} t\right), \cdots, F_{x}^{n} v\left(k_{n} x, k_{n} t\right)\right)\right)
$$

Eq.(3.9) becomes

$$
\left(F_{x^{n}}^{c}\right)_{0}+\frac{g_{x^{n-1}}}{g_{x^{n}}}\left(F_{x^{n-1}}^{c}\right)_{0}+\cdots=-\frac{g_{\epsilon}+\frac{g}{\epsilon}}{g_{x^{n}}}
$$

Eq.(3.10) can be solved by assuming some initial guess. In most cases this initial guess is the initial condition of respective problem written as $v_{0,1}(x, t)$. Therefore, the first solution of iterative process is obtained as $v_{1,1}(x, t)$. Similarly, by using Eq.(3.4) i.e. further iterations can be obtained up to $n$ iterations. These iterations can be terminated after getting a satisfactory result. In this paper only PIA $(1,1)$ will be utilized. More general algorithm of PIA $(n, m)$ can be derived by adding more number of corrected terms in Eq.(3.4) but this will increase the calculation effort as adding more terms means additional calculations and higher algebra involvement.

\section{CONVERGENCE OF PIA FOR TIME FRACTIONAL DELAY PARTIAL DIFFERENTIAL EQUATION}

In order to start studying the convergence of the method of Perturbation Iteration Algorithm, we express the approximate solutions differently.

Let $c_{0}=v_{0}, c_{n+1}=\left(v^{c}\right)_{n}$. Similarly the other iteration solutions can be written as

$$
\begin{aligned}
v_{0} & =c_{0} \\
v_{1} & =v_{0}+\left(v^{c}\right)_{0}=c_{0}+c_{1} \\
v_{2} & =v_{1}+\left(v^{c}\right)_{1}=c_{0}+c_{1}+c_{2} \\
v_{3} & =v_{2}+\left(v^{c}\right)_{2}=c_{0}+c_{1}+c_{2}+c_{3} \\
\vdots & \\
v_{n+1} & =v_{n}+\left(v^{c}\right)_{n}=c_{0}+c_{1}+c_{2}+c_{3}+\cdots+c_{n+1}=\sum_{p=0}^{n+1} c_{p}
\end{aligned}
$$

or this can be written as $v(x, t)=\lim _{n \rightarrow \infty} v_{n+1}(x, t)=\sum_{p=0}^{\infty} c_{p}$.

Banach's fixed point theorem is defined as 
Theorem 4.1. (Banach's fixed point theorem)

Consider a Banach space $B$ with a non linear mapping $N_{L}: B \rightarrow B$. Also assume that $\left\|N_{L}(x)-N_{L}\left(x^{*}\right)\right\| \leq \alpha\left\|x-x^{*}\right\|$ where $x, x^{*} \in B$ and $\alpha$ is a constant. Then $N_{L}$ has a unique fixed point. Let $x_{0} \in B$ then the sequence $x_{n+1}=N_{L}\left(x_{n}\right)$ converges to the fixed point of $N_{L}$ and $\left\|x_{m}-x_{n}\right\| \leq\left\|x_{1}-x_{0}\right\| \sum_{p=n-1}^{m-2} \alpha^{p}$

To prove the convergence of this series solution, let's derive a theorem from Banach's fixed point theorem.

Theorem 4.2. Let the series $\sum_{p=0}^{\infty} c_{p}$ be defined on Banach space B with a norm $\|\cdot\|$. Let us assume that $v_{0}=c_{0}$ be the initial guess will remain inside the ball of $v(x, t)$. The series solution $\sum_{p=0}^{\infty} c_{p}$ converges only if there exists $\alpha$ such that $\left\|c_{n+1}\right\| \leq \alpha\left\|c_{n}\right\|$

Proof:

In order to prove the convergence of the sequence defined in Eq.(4.12) we will show that this is a Cauchy sequence in $B$.

$$
\left\|v_{n+1}-v_{n}\right\|=\left\|c_{n+1}\right\| \leq \alpha\left\|c_{n}\right\| \leq \alpha^{2}\left\|c_{n-1}\right\| \leq \cdots \leq \alpha^{n+1}\left\|c_{0}\right\|
$$

For every $m, n \in N, n \geq m$ we have

$$
\begin{gathered}
\left\|v_{n}-v_{m}\right\|=\left\|\left(v_{n}-v_{n-1}\right)+\left(v_{n-1}-v_{n-2}\right)+\cdots+\left(v_{m+1}-v_{m}\right)\right\| \\
\leq\left\|v_{n}-v_{n-1}\right\|+\left\|v_{n-1}-v_{n-2}\right\|+\cdots+\left\|v_{m+1}-v_{m}\right\| \\
\leq \alpha^{n}\left\|c_{0}\right\|+\alpha^{n-1}\left\|c_{0}\right\|+\cdots+\alpha^{m+1}\left\|c_{0}\right\|
\end{gathered}
$$

Since this is a geometric series so its sum can be written as

$$
\begin{gathered}
=\frac{1-\alpha^{n-m}}{1-\alpha} \alpha^{m+1}\left\|c_{0}\right\| \\
\lim _{n, m \rightarrow \infty}\left\|v_{n}-v_{m}\right\|=\lim _{n, m \rightarrow \infty}\left(\frac{1-\alpha^{n-m}}{1-\alpha} \alpha^{m+1}\left\|c_{0}\right\|\right)=\lim _{m \rightarrow \infty}\left(\frac{\alpha^{m+1}}{1-\alpha}\left\|c_{0}\right\|\right)
\end{gathered}
$$

Also $0<\alpha<1$ therefore $\lim _{n, m \rightarrow \infty}\left\|v_{n}-v_{m}\right\|=0$. Hence it shows that its a Cauchy sequence so it is convergent.

\section{NUMERICAL EXAMPLES}

This section discusses both the reliability and efficacy of the numerical method proposed. Some numerical examples have been solved by PIA to prove that this numerical technique is an elegant and effortless method, and discussion of its findings has also been given below. These examples include three examples of TFDPDEs. Mathematica, a mathematical software, has been utilized for calculations and graphics in all three examples. 
5.1. Example. Let's consider the first example as a generalized Time Fractional Burgers Equation with proportional delay [22]

$$
D_{t}^{\alpha} v(x, t)=v_{x x}(x, t)+v(k x, k t) v_{x}(x, k t)+\frac{1}{2} v(x, t) .
$$

with initial condition $v(x, 0)=x$. Now by introducing $\epsilon$ with non linear terms in Eq.(5.14) it becomes

$$
D_{t}^{\alpha} v(x, t)=\epsilon v_{x x}(x, t)+\epsilon v(k x, k t) v_{x}(x, k t)+\frac{1}{2} v(x, t) .
$$

By applying formula in Eq.(3.8) and put $\epsilon=0$, Eq.(5.15) becomes

$$
\left(D_{t}^{\alpha} v(x, t)\right)^{c}=-D_{t}^{\alpha} v(x, t)+v_{x x}(x, t)+v(k x, k t) v_{x}(x, k t)+\frac{1}{2} v(x, t) .
$$

By using Eq.(3.8) and consider the initial guess in this case be $v_{0}(x, t)=x$ we have

$$
\begin{aligned}
v_{0}(x, t)= & x \\
v_{1}(x, t)= & x+\frac{(1+2 k) x t^{\alpha}}{2 \Gamma(\alpha+1)} \\
v_{2}(x, t)= & x+\frac{(1+2 k) x t^{\alpha}}{2 \Gamma(\alpha+1)}+\frac{(1+2 k)\left(1+4 k^{\alpha+1}\right) x t^{2 \alpha}}{4 \Gamma(2 \alpha+1)}+\frac{k^{2 \alpha+1}(1+2 k)^{2} x t^{3 \alpha} \Gamma(\alpha+1)}{4 \Gamma(\alpha+1)^{2} \Gamma(3 \alpha+1)} \\
v_{3}(x, t)= & x+\frac{(1+2 k) x t^{\alpha}}{2 \Gamma(\alpha+1)}+\frac{(1+2 k)\left(1+4 k^{\alpha+1}\right) x t^{2 \alpha}}{4 \Gamma(2 \alpha+1)}+\frac{2 k^{3 \alpha+1}(2 k+1) x t^{3 \alpha}}{\Gamma(3 \alpha+1)}+ \\
& \left(\frac{2 k^{\alpha+1}\left(2 k^{\alpha+1}+k^{\alpha}+2 k\right)}{2}+\frac{4 k^{\alpha+1}+2 k+1}{8}\right) \frac{x t^{3 \alpha}}{\Gamma(3 \alpha+1)}+ \\
& \frac{k^{2 \alpha+1}(2 k+1)^{2} x t^{3 \alpha} \Gamma(2 \alpha+1)}{4(\Gamma(\alpha+1))^{2} \Gamma(3 \alpha+1)}+\left(2 k^{5 \alpha+3}(k+1)+\frac{k^{2 \alpha+1}(4 k+1)}{8}+\right. \\
& \left.\frac{k^{2 \alpha+2}\left(k^{3 \alpha}+k\right)}{2}\right) \frac{x t^{4 \alpha} \Gamma(2 \alpha+1)}{\Gamma(\alpha+1)^{2} \Gamma(4 \alpha+1)}+\cdots
\end{aligned}
$$

At $k=\frac{1}{2}$, Eq.(5.14) has exact solution $v(x, t)=x e^{t}$. Iterations obtained for Eq.(5.14) at $k=\frac{1}{2}$ by using Eq.(3.8) are given as

$$
\begin{aligned}
v_{0}(x, t)= & x \\
v_{1}(x, t)= & x+\frac{x t^{\alpha}}{\Gamma(\alpha+1)} \\
v_{2}(x, t)= & x+\frac{x t^{\alpha}}{\Gamma(\alpha+1)}+\frac{\left(2+2^{\alpha}\right) x t^{2 \alpha}}{2^{\alpha+1} \Gamma(2 \alpha+1)}+\frac{x t^{3 \alpha}}{2^{2 \alpha+1} \Gamma(2 \alpha+1)^{2} \Gamma(3 \alpha+1)} \\
v_{3}(x, t)= & x+\frac{x t^{\alpha}}{\Gamma(\alpha+1)}+\frac{\left(2+2^{\alpha}\right) x t^{2 \alpha}}{2^{\alpha+1} \Gamma(2 \alpha+1)}+\frac{x t^{3 \alpha}}{2^{2 \alpha+1} \Gamma(2 \alpha+1)^{2} \Gamma(3 \alpha+1)}+ \\
& \frac{\left(1+2^{\alpha+1}+2^{2 \alpha+1}+2^{3 \alpha+2}\right) x t^{3 \alpha}}{2^{3 \alpha+2} \Gamma(3 \alpha+1)}+\frac{x t^{3 \alpha} \Gamma(2 \alpha+1)}{2^{\alpha+1}(\Gamma(\alpha+1))^{2} \Gamma(3 \alpha+1)}+ \\
& \frac{\left(1+2^{3 \alpha+1}\right) x t^{4 \alpha} \Gamma(2 \alpha+1)}{4^{2 \alpha+1}(\Gamma(\alpha+1))^{2} \Gamma(4 \alpha+1)}+\frac{\left(1+3 \cdot 2^{\alpha}+2^{2 \alpha+1}\right) x t^{4 \alpha} \Gamma(3 \alpha+1)}{2^{3 \alpha+2} \Gamma(\alpha+1) \Gamma(2 \alpha+1) \Gamma(4 \alpha+1)}+
\end{aligned}
$$




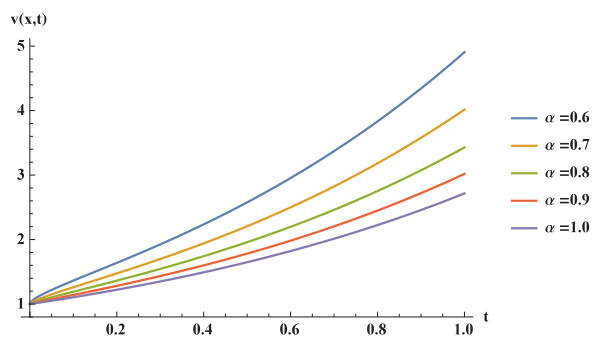

(a) Graphical representation of fractional behaviour of generalized Time-Fractional Burgers Equation in Eq.(5.14) with delay at $k=\frac{1}{2}$ and $x=1$.

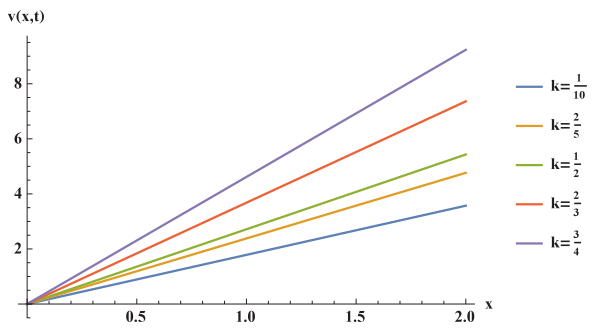

(c) Graphical representation of Eq.(5.14) with varying delay term i.e. for different values of $k$ between 0 and 1 with $t=1, \alpha=1$, which clearly shows the variation in time delay of the numerical solution.

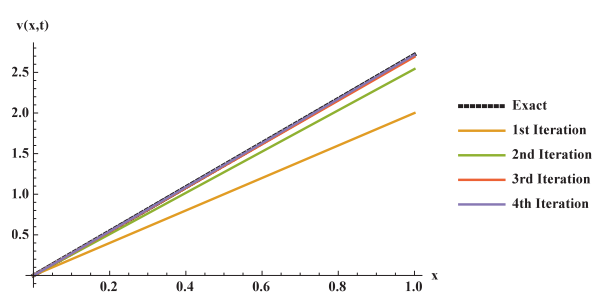

(b) Graphical representation of rate of convergence of Eq.(5.14) at $t=1, \alpha=1$ and $k=\frac{1}{2}$.

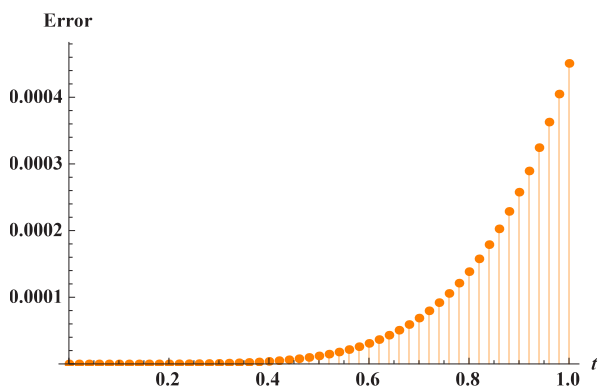

(d) Absolute error is depicted in this figure of different iterations with exact solution of Eq.(5.14) at $x=1, \alpha=1$ and $k=\frac{1}{2}$.

FIGURE 1. A detailed graphical analysis of generalized Time-Fractional Burgers Equation in Eq.(5.14) with varying delay term and its fractional behaviour is shown here. Also the fast convergence of Eq.(5.14) by PIA at $\alpha=1$ and the graph of absolute error is depicted to show the increase in accuracy of iterations of PIA.

$$
\frac{\left(1+2^{\alpha+1}\right)^{2} x t^{5 \alpha} \Gamma(4 \alpha+1)}{2^{4 \alpha+3}(\Gamma(2 \alpha+1))^{2} \Gamma(5 \alpha+1)}+\cdots
$$

After solving the generalized Time Fractional Burgers Equation by PIA numerically following observations have been made:

- PIA solved the generalized Time Fractional Burgers Equation numerically and attained a remarkable solution that is very close to the exact one. In Fig.1(b), this can be found that the third iteration is very similar to the exact solution, whereas, the fourth iteration converges absolutely to the exact solution. Estimating through further iterations convergence gets more accurate.

- The fractional behaviour of the generalized Time Fractional Burgers Equation with proportional $k=\frac{1}{2}$ was another viewpoint understudy of this work. The fractional 
behaviour of the numerical fractional solution obtained by PIA can be seen in Fig 1(a). The variance implies that since $\alpha \in[0,0.5]$ the numerical solution shows immense variability and separates itself from the actual solution, whereas for $\alpha \in$ $[0.5,1.0]$ the numerical solution has smaller changes/variation and the solution appears similar to the exact solution.

- Another aspect of this work is to study the change that occurs in the generalized Time Fractional Burgers Equation's numerical solution due to the change in the delay term value. It is found that the solution would behave divergently if $-\infty<$ $k<0$ and $1<k<+\infty$. Whereas, $k$ only appropriately responds if $k \in[0,1]$. In Fig.1(c) there emerges another observation that there is a smaller variation in the numerical solution for the $k \in[0,0.5]$ interval but a major deviation is observed for $k \in[0.5,1.0]$.

- A detailed analysis of the numerical solution obtained by PIA for $\alpha=$ $0.50,0.75,1.0$ is given in Table 1 . The $\alpha=1.0$ solution for $k=\frac{1}{2}$ is then compared with the exact solution by estimating its absolute error which validates PIA accuracy see $1(d)$.

The impact of numerical results for this first case can be clearly seen in Fig 1 and Table 1 in which the comparison among the exact solution and the numerical solution obtained by PIA at $\alpha=1.0$ and $k=\frac{1}{2}$ has complete coherence with the exact solution.

5.2. Example 2. Consider the second example of generalized Time Fractional Delay Partial Differential Equation taken from [10] as

$$
D_{t}^{\alpha} v(x, t)=v(x, k t) v_{x x}(x, k t)-v(x, t)
$$

with initial condition $v(x, 0)=x^{2}$. Now for applying PIA, identify the non linear terms and place $\epsilon$ with them, therefore Eq.(5.19) becomes

$$
D_{t}^{\alpha} v(x, t)=\epsilon v(x, k t) v_{x x}(x, k t)-v(x, t)
$$

By applying formula in Eq.(3.7) and put $\epsilon=0$, Eq.(5.20) becomes

$$
\left(D_{t}^{\alpha} v(x, t)\right)^{c}=-D_{t}^{\alpha} v(x, t)+v(x, k t) v_{x x}(x, k t)-v(x, t)
$$

By using Eq.(3.8) and consider the initial guess in this case be $v_{0}(x, t)=x^{2}$ we have

$$
\begin{aligned}
v_{0}(x, t)= & x^{2} \\
v_{1}(x, t)= & x^{2}+\frac{x^{2} t^{\alpha}}{\Gamma(\alpha+1)} \\
v_{2}(x, t)= & x^{2}+\frac{x^{2} t^{\alpha}}{\Gamma(\alpha+1)}+\frac{\left(4 k^{\alpha}-1\right) x^{2} t^{2 \alpha}}{\Gamma(2 \alpha+1)}+\frac{2 k^{2 \alpha} x^{2} t^{3 \alpha} \Gamma(2 \alpha+1)}{\Gamma(\alpha+1)^{2} \Gamma(3 \alpha+1)} \\
v_{3}(x, t)= & x^{2}+\frac{x^{2} t^{\alpha}}{\Gamma(\alpha+1)}+\frac{\left(4 k^{\alpha}-1\right) x^{2} t^{2 \alpha}}{\Gamma(2 \alpha+1)}+\frac{\left(16 k^{3 \alpha}-4 k^{2 \alpha}-4 k^{\alpha}+1\right) x^{2} t^{3 \alpha}}{\Gamma(3 \alpha+1)}+ \\
& \frac{2 k^{2 \alpha} x^{2} t^{3 \alpha} \Gamma(2 \alpha+1)}{\Gamma(\alpha+1)^{2} \Gamma(3 \alpha+1)}+\frac{2 k^{2 \alpha}\left(4 k^{3 \alpha}-1\right) x^{2} t^{4 \alpha} \Gamma(2 \alpha+1)}{\Gamma(\alpha+1)^{2} \Gamma(4 \alpha+1)}+ \\
& \frac{4 k^{3 \alpha}\left(4 k^{\alpha}-1\right) x^{2} t^{4 \alpha} \Gamma(3 \alpha+1)}{\Gamma(\alpha+1) \Gamma(2 \alpha+1) \Gamma(4 \alpha+1)}+\frac{2 k^{4 \alpha}\left(1-4 k^{\alpha}\right) x^{2} t^{5 \alpha} \Gamma(4 \alpha+1)}{\Gamma(2 \alpha+1)^{2} \Gamma(5 \alpha+1)}+
\end{aligned}
$$




$$
\frac{4 k^{3 \alpha}\left(4 k^{\alpha}-1\right) x^{2} t^{4 \alpha} \Gamma(3 \alpha+1)}{\Gamma(\alpha+1) \Gamma(2 \alpha+1) \Gamma(4 \alpha+1)}+\frac{2 k^{4 \alpha}\left(1-4 k^{\alpha}\right)^{2} x^{2} t^{5 \alpha} \Gamma(4 \alpha+1)}{\Gamma(3 \alpha+1)^{2} \Gamma(5 \alpha+1)}+\cdots
$$

At $k=\frac{1}{2}$, Eq.(5.19) has exact solution $v(x, t)=x^{2} e^{t}$. Therefore the iterations obtained at $k=\frac{1}{2}$ by using Eq.(3.8) are given as

$$
\begin{aligned}
v_{0}(x, t)= & x^{2} \\
v_{1}(x, t)= & x^{2}+\frac{x^{2} t^{\alpha}}{\Gamma(\alpha+1)} \\
v_{2}(x, t)= & x^{2}+\frac{x^{2} t^{\alpha}}{\Gamma(\alpha+1)}+\frac{\left(4-2^{\alpha}\right) x^{2} t^{2 \alpha}}{2^{\alpha} \Gamma(2 \alpha+1)}+\frac{x^{2} t^{3 \alpha} \Gamma(2 \alpha+1)}{2^{2 \alpha-1} \Gamma(\alpha+1)^{2} \Gamma(3 \alpha+1)} \\
v_{3}(x, t)= & x^{2}+\frac{x^{2} t^{\alpha}}{\Gamma(\alpha+1)}+\frac{\left(4-2^{\alpha}\right) x^{2} t^{2 \alpha}}{2^{\alpha} \Gamma(2 \alpha+1)}+\frac{\left(16-2^{\alpha+2}-2^{2 \alpha+2}+2^{3 \alpha}\right) x^{2} t^{3 \alpha}}{2^{3 \alpha} \Gamma(3 \alpha+1)}+ \\
& \frac{x^{2} t^{3 \alpha} \Gamma(2 \alpha+1)}{2^{2 \alpha-1}(\Gamma(\alpha+1))^{2} \Gamma(3 \alpha+1)}+\frac{\left(2^{3 \alpha}-4\right) x^{2} t^{4 \alpha} \Gamma(2 \alpha+1)}{2^{5 \alpha-1}(\Gamma(\alpha+1))^{2} \Gamma(4 \alpha+1)}+ \\
& \frac{\left(2^{\alpha}-4\right) x^{2} t^{4 \alpha} \Gamma(3 \alpha+1)}{2^{4 \alpha-2} \Gamma(\alpha+1) \Gamma(2 \alpha+1) \Gamma(4 \alpha+1)}+\frac{\left(2^{\alpha}-4\right) x^{2} t^{5 \alpha} \Gamma(4 \alpha+1)}{2^{5 \alpha-1}(\Gamma(\alpha+1))^{2} \Gamma(5 \alpha+1)}+\cdots
\end{aligned}
$$

Following observations were made after solving Eq.(5.19) of TFDPDE by PIA was numerically solved:

- Eq.(5.19) of TFDPDE was numerically solved by PIA and obtained a spectacular solution that is very similar to the exact solution. In Fig.2(c), it is easy to see that the second iteration is very close to the exact solution, while the third iteration completely converges to it. By estimating through more iterations the convergence becomes more precise.

- Another aspect understudy of this work was the fractional behaviour of the second TFDPDE equation with a relative delay term i.e., $k=\frac{1}{2}$. The numerical fractional solution obtained by PIA's fractional approach can be seen in Fig.2(a), The variability implies that since $\alpha \in[0,0.5]$ the numerical solution displays immense variation and separates itself from the actual solution, whereas for $\alpha \in[0.5,1.0]$ the numerical solution has smaller changes or variations and the solution appears to be close to the exact solution.

- The key element of this analysis is the study of the difference that happens in the second TFDPDE numeric solution due to the change in the delay term value. It is observed that if $-\infty<k<0$ and $1<k<+\infty$ the solution will behave divergently. Whereas $k$ only reacts adequately if $k \in[0,1]$. Fig 2(b) also provides another remark that for the $k \in[0,0.5]$ interval there is a minor variability in the numerical solution but a large difference for $k \in[0.5,1.0]$ is observed.

- A comprehensive analysis is given in Table 2 of the numerical solution obtained by PIA for $\alpha=0.50,0.75,1.0$ for second example of TFDPDE. The solution for $\alpha=1.0$ for $k=\frac{1}{2}$ is then compared to the exact solution by estimating its absolute error which validates the accuracy of PIA see 2(d) 
TABLE 1. Numerical results of Example 5.1 for different values of $\alpha$ is depicted in this table at $k=\frac{1}{2}$. Comparison of numerical results by PIA and exact solution at $\alpha=1$ is given and its absolute error is also calculated to prove the efficiency of PIA for TFDPDE.

\begin{tabular}{|c|c|c|c|c|c|c|}
\hline $\mathrm{x}$ & $\mathrm{t}$ & $\alpha=0.50$ & $\alpha=0.75$ & $\alpha=1.0$ & Exact & Abs. Error \\
\hline \multirow{5}{*}{0.25} & 0.25 & 0.522684436 & 0.378129516 & 0.321005776 & 0.321006354 & $5.784897266 \mathrm{E}-07$ \\
& 0.50 & 0.795382335 & 0.517898593 & 0.412160318 & 0.412180318 & $1.999953540 \mathrm{E}-05$ \\
& 0.75 & 1.143456970 & 0.695505884 & 0.529085610 & 0.529250004 & $1.643943956 \mathrm{E}-05$ \\
& 1.00 & 1.587005284 & 0.923646492 & 0.678819168 & 0.679570457 & $7.512889852 \mathrm{E}-04$ \\
\hline \multirow{4}{*}{0.50} & 0.25 & 1.045368872 & 0.756259032 & 0.642011551 & 0.642012708 & $1.156979453 \mathrm{E}-06$ \\
& 0.50 & 1.590764670 & 1.035797186 & 0.824320635 & 0.824360635 & $3.999990706 \mathrm{E}-05$ \\
& 0.75 & 2.286913940 & 1.391011769 & 1.058171220 & 1.058500008 & $3.287887971 \mathrm{E}-04$ \\
& 1.00 & 3.174010569 & 1.847292985 & 1.357638336 & 1.359140914 & $1.502577970 \mathrm{E}-04$ \\
\hline \multirow{5}{*}{0.75} & 0.25 & 1.568053309 & 1.134388548 & 0.963017327 & 0.963019063 & $1.735469180 \mathrm{E}-06$ \\
& 0.50 & 2.386147005 & 1.553695778 & 1.236480953 & 1.236540953 & $5.999986059 \mathrm{E}-05$ \\
& 0.75 & 3.430370911 & 2.086517653 & 1.587256829 & 1.587750012 & $4.931831867 \mathrm{E}-04$ \\
& 1.00 & 4.761015853 & 2.770939477 & 2.036457504 & 2.038711371 & $2.253866955 \mathrm{E}-03$ \\
\hline \multirow{4}{*}{1.00} & 0.25 & 2.090737745 & 1.512518064 & 1.284023103 & 1.284025417 & $2.313958910 \mathrm{E}-06$ \\
& 0.50 & 3.181529340 & 2.071594371 & 1.648641271 & 1.648721271 & $7.999981412 \mathrm{E}-05$ \\
& 0.75 & 4.573827881 & 2.782023537 & 2.116342439 & 2.117000017 & $6.575775822 \mathrm{E}-04$ \\
& 1.00 & 6.348021138 & 3.694585970 & 2.715276673 & 2.718281828 & $3.005155941 \mathrm{E}-03$ \\
\hline
\end{tabular}

TABLE 2. Numerical results of Example 5.2 for different values of $\alpha$ is depicted in this table at $k=\frac{1}{2}$. Comparison of numerical results by PIA and exact solution at $\alpha=1$ is given and its absolute error is also calculated to prove the efficiency of PIA for TFDPDE.

\begin{tabular}{|c|c|c|c|c|c|c|}
\hline $\mathrm{x}$ & $\mathrm{t}$ & $\alpha=0.50$ & $\alpha=0.75$ & $\alpha=1.0$ & Exact & Abs. Error \\
\hline \multirow{5}{*}{0.25} & 0.25 & 0.163526842 & 0.096882628 & 0.080251575 & 0.080251589 & $1.384959599 \mathrm{E}-08$ \\
& 0.50 & 0.329265720 & 0.138303839 & 0.103044200 & 0.103045079 & $8.794403900 \mathrm{E}-07$ \\
& 0.75 & 0.636665410 & 0.195505780 & 0.132302605 & 0.132312501 & $9.896096527 \mathrm{E}-06$ \\
& 1.00 & 1.181668546 & 0.275777661 & 0.169837966 & 0.169892614 & $5.464804536 \mathrm{E}-05$ \\
\hline \multirow{5}{*}{0.50} & 0.25 & 0.654107367 & 0.387530513 & 0.321006299 & 0.321006354 & $5.539838399 \mathrm{E}-08$ \\
& 0.50 & 1.317062879 & 0.553215357 & 0.412176800 & 0.412180318 & $3.517761558 \mathrm{E}-06$ \\
& 0.75 & 2.546661640 & 0.782023121 & 0.529210420 & 0.529250004 & $3.958438610 \mathrm{E}-05$ \\
& 1.00 & 4.726674183 & 1.103110644 & 0.679351865 & 0.679570457 & $2.185921814 \mathrm{E}-04$ \\
\hline \multirow{5}{*}{0.75} & 0.25 & 1.471741577 & 0.871943654 & 0.722264172 & 0.722264297 & $1.246463640 \mathrm{E}-07$ \\
& 0.50 & 2.963391478 & 1.244734553 & 0.927397800 & 0.927405715 & $7.914963506 \mathrm{E}-06$ \\
& 0.75 & 5.729988691 & 1.759552022 & 1.190723444 & 1.190812509 & $8.906486874 \mathrm{E}-05$ \\
& 1.00 & 10.635016911 & 2.481998948 & 1.528541696 & 1.529033529 & $4.918324083 \mathrm{E}-04$ \\
\hline \multirow{1}{*}{1.00} & 0.25 & 2.616429470 & 1.550122051 & 1.284025195 & 1.284025417 & $2.215935399 \mathrm{E}-07$ \\
& 0.50 & 5.268251517 & 2.212861428 & 1.648707200 & 1.648721271 & $1.407104623 \mathrm{E}-05$ \\
& 0.75 & 10.186646561 & 3.128092484 & 2.116841679 & 2.117000017 & $1.583375444 \mathrm{E}-04$ \\
& 1.00 & 18.906696731 & 4.412442574 & 2.717407460 & 2.718281828 & $8.743687258 \mathrm{E}-04$ \\
\hline
\end{tabular}




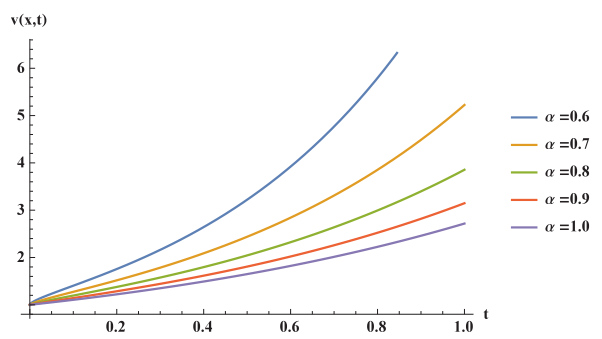

(a) Graphical representation of numerical solution of Example 5.2 is shown here for different values of $\alpha$ with $x=1$ and $k=\frac{1}{2}$. It depicts the fractional behavior and the variation occuring in the numerical solution due to the change in the value of $\alpha$.

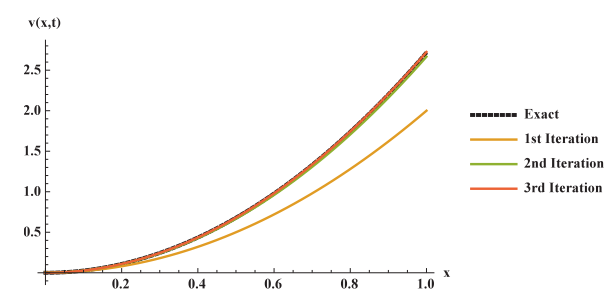

(b) Graphical verification of fast rate of convergence of Example 5.2 of TFDPDE where $k=\frac{1}{2}, \alpha=1$ and $t=1$.
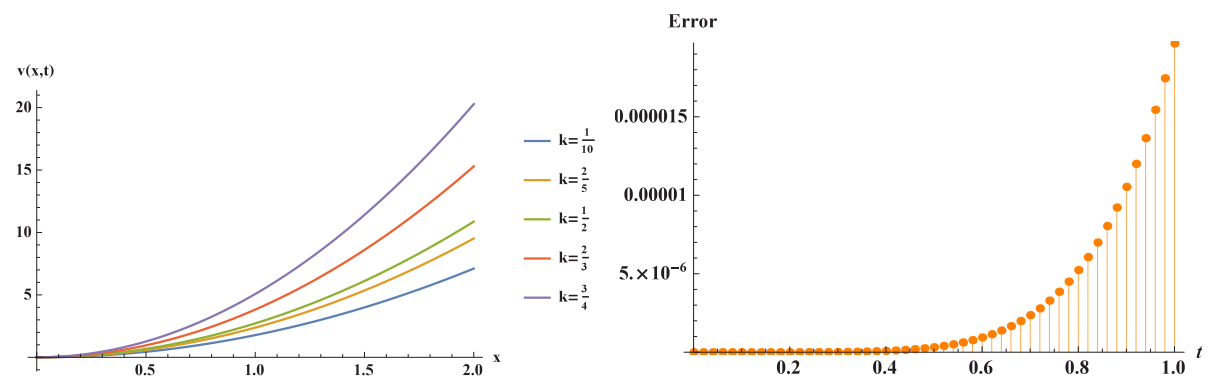

(c) Graphical representation of Eq. (5.19) with vary- (d) Absolute error is depicted in this figure of difing delay term i.e. for different values of $k$ between ferent iterations with exact solution of Eq.(5.19) at 0 and 1 with $t=1, \alpha=1$, which clearly shows the $x=1, \alpha=1$ and $k=\frac{1}{2}$.

variation in time delay of the numerical solution.

FIGURE 2. A detailed graphical analysis of TFDPDE in Eq.(5.19) with varying delay term and its fractional behaviour is shown here. Also the fast convergence of Eq.(5.19) by PIA at $\alpha=1$ and the graph of absolute error is depicted to show the increase in accuracy of iterations of PIA.

For this second case, the significance of the numerical results can be evidently seen in Fig 2 and Table 2, in which the comparison between the exact solution and the numerical solution obtained by PIA at $\alpha=1.0$ and $k=\frac{1}{2}$ is fully consistent with the exact solution.

5.3. Example 3. Let the third example of generalized Time Fractional Delay Partial Differential Equation be taken from [22] as

$$
D_{t}^{\alpha} v(x, t)=u_{x x}(k x, k t) v_{x}(k x, k t)-\frac{1}{8} v_{x}(x, t)-v(x, t)
$$

with initial condition $v(x, 0)=x^{2}$. Now to apply PIA, let's first introduce $\epsilon$ with non linear terms in Eq.(5.24) as

$$
D_{t}^{\alpha} v(x, t)=\epsilon u_{x x}(k x, k t) v_{x}(k x, k t)-\epsilon \frac{1}{8} v_{x}(x, t)-v(x, t)
$$


By applying formula in Eq.(3.7) and put $\epsilon=0$, Eq.(5.25) becomes

$$
\left(D_{t}^{\alpha} v(x, t)\right)^{c}=-D_{t}^{\alpha} v(x, t)+u_{x x}(k x, k t) v_{x}(k x, k t)-\frac{1}{8} v_{x}(x, t)-v(x, t)
$$

therefore by using the initial guess $v_{0}(x, t)=x^{2}$ and Eq.(3.8), we obtain the following iterations as

$$
\begin{aligned}
v_{0}(x, t)= & x^{2} \\
v_{1}(x, t)= & x^{2}+\frac{\left(16 k^{4}-1\right) x t^{\alpha}}{4 \Gamma(\alpha+1)}-\frac{x^{2} t^{\alpha}}{\Gamma(\alpha+1)} \\
v_{2}(x, t)= & x^{2}-\frac{\left(16 k^{4}+1\right) x t^{\alpha}}{4 \Gamma(\alpha+1)}-\frac{x^{2} t^{\alpha}}{\Gamma(\alpha+1)}+\frac{\left(16 k^{4}-1\right)\left(16 k^{\alpha+3}-1\right) t^{3 \alpha}}{32 \Gamma(2 \alpha+1)}- \\
& \frac{\left(16 k^{\alpha+4}+8 k^{4}-1\right) x t^{2 \alpha}}{2 \Gamma(2 \alpha+1)}+\frac{x^{2} t^{2 \alpha}}{\Gamma(2 \alpha+1)}-\frac{k^{2 \alpha+3}\left(16 k^{4}-1\right) t^{3 \alpha} \Gamma(2 \alpha+1)}{2 \Gamma(\alpha+1)^{2} \Gamma(3 \alpha+1)}+ \\
& \frac{4 k^{2 \alpha+4} x t^{3 \alpha} \Gamma(2 \alpha+1)}{\Gamma(\alpha+1)^{2} \Gamma(3 \alpha+1)} \\
v_{3}(x, t)= & x^{2}-\frac{\left(16 k^{4}+1\right) x t^{\alpha}}{4 \Gamma(\alpha+1)}-\frac{x^{2} t^{\alpha}}{\Gamma(\alpha+1)}+\frac{\left(16 k^{4}-1\right)\left(16 k^{\alpha+3}-1\right) t^{3 \alpha}}{32 \Gamma(2 \alpha+1)}- \\
& \frac{\left(32 k^{4}+3\right) T^{3 \alpha}}{32 \Gamma(3 \alpha+1)}+\frac{k^{\alpha+3}\left(16 k^{2 \alpha+4}+8 k^{\alpha+4}-k^{\alpha}-8 k^{4}\right) t^{3 \alpha}}{\Gamma(3 \alpha+1)}- \\
& \frac{\left(32 k^{2 \alpha+4}+32 k^{\alpha+4}+16 k^{4}+3\right) x t^{3 \alpha}}{4 \Gamma(3 \alpha+1)}-\frac{x^{2} t^{3 \alpha}}{\Gamma(3 \alpha+1)}- \\
& \frac{k^{2 \alpha+3}\left(16 k^{4}-1\right) t^{3 \alpha} \Gamma(2 \alpha+1)}{2 \Gamma(\alpha+1)^{2} \Gamma(3 \alpha+1)}+\frac{4 k^{2 \alpha+4} x t^{3 \alpha} \Gamma(2 \alpha+1)}{\Gamma(\alpha+1)^{2} \Gamma(3 \alpha+1)}- \\
& \frac{k^{2 \alpha+3} t^{4 \alpha} \Gamma(2 \alpha+1)}{2 \Gamma(\alpha+1)^{2} \Gamma(4 \alpha+1)}-\cdots
\end{aligned}
$$

At $k=\frac{1}{2}$, its exact solution is $v(x, t)=x^{2} e^{-t}$. Therefore the iterations obtained at $k=\frac{1}{2}$ by using Eq.(3.8) are given as

$$
\begin{aligned}
v_{0}(x, t)= & x^{2} \\
v_{1}(x, t)= & x^{2}-\frac{x^{2} t^{\alpha}}{\Gamma(\alpha+1)} \\
v_{2}(x, t)= & x^{2}-\frac{x^{2} t^{\alpha}}{\Gamma(\alpha+1)}+\frac{\left(-2+2^{\alpha}+2^{\alpha+2} x\right) x t^{2 \alpha}}{2^{\alpha+2} \Gamma(2 \alpha+1)}+\frac{x t^{3 \alpha} \Gamma(2 \alpha+1)}{2^{2 \alpha+2} \Gamma(\alpha+1)^{2} \Gamma(3 \alpha+1)} \\
v_{3}(x, t)= & x^{2}-\frac{x^{2} t^{\alpha}}{\Gamma(\alpha+1)}+\frac{\left(-2+2^{\alpha}+2^{\alpha+2} x\right) x t^{2 \alpha}}{2^{\alpha+2} \Gamma(2 \alpha+1)}+\frac{\left(-4+2^{\alpha+1}+2^{2 \alpha+1}+{ }^{3 \alpha}\right) t^{3 \alpha}}{2^{3 \alpha+5} \Gamma(3 \alpha+1)}- \\
& \frac{\left(-1-2^{\alpha}+2^{2 \alpha}+2^{2 \alpha+1} x\right) x t^{3 \alpha}}{2^{2 \alpha+1} \Gamma(3 \alpha+1)}+\frac{x t^{3 \alpha} \Gamma(2 \alpha+1)}{2^{2 \alpha+2}(\Gamma(\alpha+1))^{2} \Gamma(3 \alpha+1)}+ \\
& \frac{\left(2-2^{3 \alpha}-2^{3 \alpha+3} x\right) t^{4 \alpha} \Gamma(2 \alpha+1)}{2^{5 \alpha+5}(\Gamma(\alpha+1))^{2} \Gamma(4 \alpha+1)}+\frac{\left(2-2^{\alpha}-2^{\alpha+3} x\right) t^{4 \alpha} \Gamma(3 \alpha+1)}{2^{4 \alpha+4} \Gamma(\alpha+1) \Gamma(2 \alpha+1) \Gamma(4 \alpha+1)}+\cdots
\end{aligned}
$$




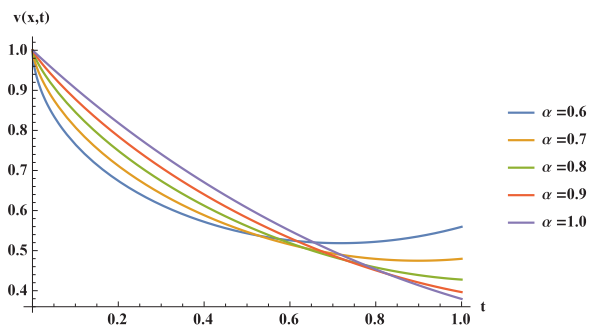

(a) Fractional behavior of the numerical solution of Example 5.3 of TFDPDE for different values of $\alpha$ for $x=1$ and $k=\frac{1}{2}$ is depicted here.

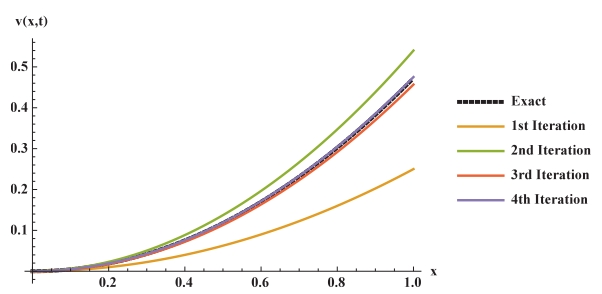

(b) Graphical verification of fast rate of convergence of Example 5.3 of TFDPDE where $k=\frac{1}{2}, \alpha=1$ and $t=1$.
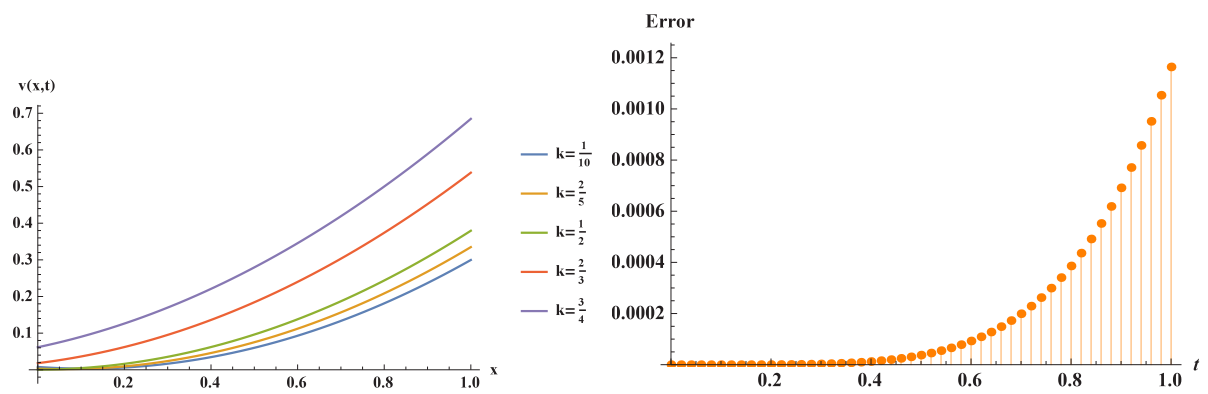

(c) Graphical representation of Eq.(5.24) with vary- (d) Absolute error is depicted in this figure of difing delay term i.e. for different values of $k$ between ferent iterations with exact solution of Eq.(5.24) at 0 and 1 with $t=1, \alpha=1$, which clearly shows the $x=1, \alpha=1$ and $k=\frac{1}{2}$.

variation in time delay of the numerical solution.

FIGURE 3. A detailed graphical analysis of TFDPDE in Eq.(5.24) with varying delay term and its fractional behaviour is shown here. Also the fast convergence of Eq.(5.24) by PIA at $\alpha=1$ and the graph of absolute error is depicted to show the increase in accuracy of iterations of PIA.

After solving the third example of TFDPDE by PIA numerically following observations have been made:

- Eq.(5.24) of TFDPDE solved by PIA numericallyattained a solution that is very close to the exact one. This can be observed in Fig 3(c) that the third iteration is very similar to the exact solution, while the fourth iteration absolutely converges to it, as seen before. The convergence becomes more accurate by estimating further iterations.

- Another perspective is the fractional behaviour of Eq.(5.24) of TFDPDE with proportional $k=\frac{1}{2}$. The numerical fractional solution obtained by PIA's fractional behaviour can be seen in Fig.3(a). Such variations suggest that since $\alpha \in[0.5,1.0]$ the numerical solution shows considerable variation and distances itself from the actual solution, whereas for $\alpha \in[0,0.5]$ the numerical solution contains smaller changes or deviations and the solution tends to be close to the exact solution. 
TABLE 3. Numerical results of Example 5.3 for different values of $\alpha$ is depicted in this table at $k=\frac{1}{2}$. Comparison of exact solution and numerical results by PIA at $\alpha=1$ is given and its absolute error is also calculated to prove the efficiency of PIA for TFDPDE.

\begin{tabular}{|c|c|c|c|c|c|c|}
\hline $\mathrm{x}$ & $\mathrm{t}$ & $\alpha=0.50$ & $\alpha=0.75$ & $\alpha=1.0$ & Exact & Abs. Error \\
\hline \multirow{5}{*}{0.25} & 0.25 & 0.0367870479 & 0.042746073 & 0.048676973 & 0.048675049 & $1.924270395 \mathrm{E}-06$ \\
& 0.50 & 0.0346486403 & 0.033868319 & 0.037968542 & 0.037908166 & $6.037561727 \mathrm{E}-05$ \\
& 0.75 & 0.0409965441 & 0.030051332 & 0.029972537 & 0.029522910 & $4.496271106 \mathrm{E}-04$ \\
& 1.00 & 0.0557130964 & 0.031832167 & 0.024850978 & 0.022992465 & $1.858512971 \mathrm{E}-03$ \\
\hline \multirow{5}{*}{0.50} & 0.25 & 0.1513393957 & 0.172291371 & 0.194704644 & 0.194700196 & $4.448647287 \mathrm{E}-06$ \\
& 0.50 & 0.1387070622 & 0.137226997 & 0.151771334 & 0.151632665 & $1.386686983 \mathrm{E}-04$ \\
& 0.75 & 0.1503810962 & 0.119012382 & 0.119117951 & 0.118091638 & $1.026313216 \mathrm{E}-03$ \\
& 1.00 & 0.1836818353 & 0.116612028 & 0.096187319 & 0.091969860 & $4.217458277 \mathrm{E}-03$ \\
\hline \multirow{5}{*}{0.75} & 0.25 & 0.3437703459 & 0.388662982 & 0.438083390 & 0.438075440 & $7.949359004 \mathrm{E}-06$ \\
& 0.50 & 0.3129097241 & 0.310255991 & 0.341420480 & 0.341173496 & $2.469834818 \mathrm{E}-04$ \\
& 0.75 & 0.3304459565 & 0.267557562 & 0.267528503 & 0.265706186 & $1.822316947 \mathrm{E}-03$ \\
& 1.00 & 0.3890715811 & 0.256214767 & 0.214398659 & 0.206932186 & $7.466473437 \mathrm{E}-03$ \\
\hline \multirow{3}{*}{1.00} & 0.25 & 0.6140798985 & 0.691860907 & 0.778813209 & 0.778800783 & $1.242640554 \mathrm{E}-05$ \\
& 0.50 & 0.5572566258 & 0.552955301 & 0.606915980 & 0.606530660 & $3.853199680 \mathrm{E}-04$ \\
& 0.75 & 0.5811911247 & 0.475686873 & 0.475204191 & 0.472366553 & $2.837638304 \mathrm{E}-03$ \\
& 1.00 & 0.6718823337 & 0.450640385 & 0.379485000 & 0.367879441 & $1.160555845 \mathrm{E}-02$ \\
\hline
\end{tabular}

- Another aspect of this work is to study the change that happens in Eq.(5.24) of TFDPDE's numerical solution due to the change in the delay term value. It is found that the solution would behave divergently if $-\infty<k<0$ and $1<k<$ $+\infty$. Whereas $k$ only appropriately responds if $k \in[0,1]$. Fig. 3(b) also makes another observation that there is a smaller variation in the numerical solution for the $k \in[0,0.5]$ interval but a major deviation is observed for $k \in[0.5,1.0]$.

- A detailed analysis of the numerical solution obtained by PIA for $\alpha=$ $0.50,0.75,1.0$ is given in Table 3. The $\alpha=1.0$ solution for $k=\frac{1}{2}$ is then compared with the exact solution by estimating its absolute error which validates PIA accuracy see 3(d)

For this third case, the effect of the numerical results can be simply seen in Fig 3 and Table 3, in which the comparison between the exact solution and the numerical solution obtained by PIA at $\alpha=1.0$ and $k=\frac{1}{2}$ is entirely compatible with the exact solution.

\section{CONCLUSION}

The prime purpose of this research paper was to relate two physical effects for partial differential equations on the time-coordinate, notably the multiple-delay times and fractional-derivative. A different type of the numerical method relying on the Taylor series was employed to find the proposed model's approximated analytical solutions and the following scientific facts were drawn: 
- Numerical algorithm shows that the mathematical strategy exhibits the necessary convergence orders very well in line with our theoretical analysis see Table $1 / 2 \mid 3$ and Fig 1(b), 2(b), 3(b).

- Ultimately, the resulting solutions to these problems have an asymptotic continuous aspect when the order of the fractional derivative is changed a bit see Fig. 1(a), 2(a), 3(a)

- Delay term should always be considered for $k \in[0,1]$. For the remaining values on the number line i.e. $-\infty<k<0$ and $1<k<+\infty$ the numerical solution either shows no delay or divergent solution which insists to consider $k \in[0,1]$ see Fig. 1(c), 2(c), 3(c),

- It can also be proposed for different forms of fractional derivatives. For most forms, the principle in differentiating the power function is the same.

- Less computing work is required with efficient accuracy.

- In order to determine the components of the solution with high speed execution time, it leads directly to an iterative scheme.

- Fractional series solution is more stable while fractional order values $\alpha$ approach 1 see Fig. 1(a), 2(a), 3(a),

- Behaviour of $k$ is clearly observed in these examples as $k$ ranges between $[0.5,1.0]$ the numerical solution shows higher in variation whereas $[0,0.5], k$ shows less variation and closer to the exact solution.

\section{ACKNOWLEDGE}

The authors are grateful for the comments of anonymous referees, who have helped improve the manuscript qualitatively, and are deeply in debt to the editor for his enlightening advice and useful suggestions.

\section{REFERENCES}

[1] I. Abu-Irwaq, M. Alquran, I. Jaradat, M.S.M. Noorani, S. Momani and D. Baleanu, Numerical investigations on the physical dynamics of the coupled fractional Boussinesq-Burgers system, Romanian Journal of Physics, 65, No.5-6 (2020) 111.

[2] M. Ali, M. Alquran and I. Jaradat, Asymptotic-sequentially solution style for the generalized Caputo timefractional NewellWhiteheadSegel system, Adv Differ Equ, 2019 (2019) 2-9. https://doi.org/10.1186/s13662019-2021-8

[3] M. Alquran, I. Jaradat, S. Momani and D. Baleanu, Chaotic and solitonic solutions for a new time-fractional two-mode Korteweg-de Vries equation, Romanian Reports in Physics, 72, No.3 (2020) 117.

[4] M. Alquran1, I. Jaradat1, M. Ali and A. Abu-Aljazar, Computational Scheme for the Time-Fractional Reaction-Diffusion Brusselator Model, International Journal of Applied and Computational Mathematics, 6, No.5 (2020) 1-10.

[5] M. Alquran, M. Ali, M. Alsukhour and I. Jaradat, Promoted residual power series technique with Laplace transform to solve some time-fractional problems arising in physics, Results in Physics, 19 (2020) 103667.

[6] M. Alquran, I. Jaradat, D. Baleanu and M. Syam, The Duffing model endowed with fractional time derivative and multiple pantograph time delays, Romanian Journal of Physics, 64, No.5-6 (2019) 107.

[7] M. Alquran and I. Jaradat, Delay-asymptotic solutions for the time-fractional delay-type wave equation, Physica A: Statistical Mechanics and its Applications, 527 (2019) 121275. https://doi.org/10.1016/j.physa.2019.121275.

[8] S.N. Busenberg and J.M. Mahaffy, Interaction of spatial diffusion and delays in models of genetic control by repression, J Math Biol, 22 (1985) 313333. 
[9] D.S. Cohen, P.S. Hagan and H.C. Simpson, Spatial structures in predator-prey communities with hereditary effects and diffusion, Math Biosci, 44 (1979) 167177.

[10] D. Durgun and K. Ali K, Fractional VIM for time fractional nonlinear functional PDEs having proportional delays, Thermal Science, 22 (2017) 269-269.

[11] K. Diethelm, Analysis of Fractional Differential Equations, Springer-Verlag, Berlin, 2010.

[12] P.K. Gupta and M. Singh, Homotopy perturbation method for fractional Fornberg Whitham equation, Computers \& Mathematics with Applications, 61, No.2 (2011) 250-254.

[13] M. Gyllenberg and H.J.A. Heijmans, An abstract delay-differential equation modelling size dependent cell growth and division, SIAM J Math Anal, 18 (1987) 7488.

[14] I. Jaradat, M. Alquran, S. Momani and D. Baleanu, Numerical schemes for studying biomathematics model inherited with memory-time and delay-time, Alexandria Engineering Journal, 59, No.5 (2020) 2969-2974.

[15] M. Khalid, M. Sultana and F.S. Khan, Numerical Solution of SIR Model of Dengue Fever, International Journal of Computer Applications, 118, No.21 (2015) 1-4.

[16] M. Khalid, F. S. Khan, H. Zehra and M. Shoaib, A Highly Accurate Numerical Method for Solving TimeFractional Partial Differential Equation, Progress in Fractional Differentiation and Applications An International Journal, 2, No.3 (2016) 227-232.

[17] M. Khalid, F.S. Khan and A. Iqbal, Perturbation-Iteration Algorithm to Solve Fractional Giving Up Smoking Mathematical Model, International Journal of Computer Applications, 142, No.9 (2016) 1-6.

[18] M. Khalid, F.S. Khan and M. Sultana, A highly accurate numerical method for solving nonlinear time-fractional differential difference equation, Math Meth Appl Sci., (2019) 111. https://doi.org/10.1002/mma.5883

[19] A.A. Kilbas, H.M. Srivastava and J.J. Trujillo, Theory and applications of fractional differential equations, Elsevier Science \& Technology, 2006.

[20] J.M. Mahaffy and C.V. Pao, Models of genetic control by repression with time delays and spatial effects, J Math Biol, 20 (1984) 3957.

[21] A.L. Mehauté, J.A.T. Machado, J.C. Trigeassou and J. Sabatier, Fractional differentiation and its applications, Proceedings of the 1st IFAC Workshop on Fractional Differentiation and Its Applications (FDA 04), ENSEIRB, 1 (2004) 353358.

[22] G.S. Mehmet, U. Fatih and E. Fevzi, Numerical solution of time-fractional nonlinear PDEs with proportional delays by homotopy perturbation method, Applied Mathematical Modelling, 40 (2016) 6639-6649.

[23] J.D. Murray, Spatial structures in predator-prey comunitiesa nonlinear time delay diffusional model, Math Biosci, 30 (1976) 7385

[24] Z. Ouyang, Existence and uniqueness of the solutions for a class of nonlinear fractional order partial differential equations with delay, Computers \& Mathematics with Applications, 61, No.4 (2011) 860-870.

[25] I. Podlubny, Fractional Differential Equations, Academic Press, New York, 1999.

[26] A.O. Rey and M.C. Mackey, Multistability and boundary layer development in a transport equation with delayed arguments, Can Appl Math, Quar 1 (1993) 121.

[27] L.I. Tingyue, Z. Qifeng, N. Wwhidullah, X. Yinghong and R. Maohua R, Effective Algorithm for Delay Fractional Convection-Diffusion Wave Equation Based on Reversible Exponential Recovery Method, IEEE Access, 7 (2019) 5554-5563. Doi:10.1109/ACCESS.2018.2889735

[28] P.K. Wang, Optimal control of parabolic systems with boundary conditions involving time delays, SIAM J Control, 13 (1975) 274293.

[29] J. Wu, Theory and Applications of Partial Functional Differential Equations, Springer-Verlag, New York, USA, 1996.

[30] Y. Yan and C. Kou, Stability analysis for a fractional differential model of HIV infection of CD4 ${ }^{+}$T-cells $^{-}$ with time delay, Mathematics \& Computers in Simulation, 82, No.9 (2012) 1572-1585.

[31] X. Zhang, Some results of linear fractional order time-delay system, Applied Mathematics and Computation, 197, No.1 (2008) 407-411. 